\title{
Simulation of Field Emission Microtriodes
}

\author{
Ernest G. Zaidman
}

\begin{abstract}
Vacuum microtriode RF amplifier performance, based upon a unit cell with a conical field emitter tip, gate, and anode, was evaluated via computer simulation. Electron emission was calculated from the Fowler-Nordheim equation. The dependence of emitted current, transconductance, and field enhancement upon geometrical factors, e.g., tip sharpness, tip height, cone half-angle, and gate hole radius, is shown. The device design parameters of transconductance, cutoff frequency, small signal gain, and efficiency, have been calculated. Electron streamlines and current flux are shown for time-dependent RF input. Because a compact electron beam source has wide application, the normalized beam emittance, brightness, and beam quality are calculated for a typical case. Potential difficulties with anode power deposition are noted.
\end{abstract}

\section{INTRODUCTION}

M ICROMETER-SCALE vacuum electronics [1] constructed by solid-state fabrication techniques is attracting increased interest [2]. Currently the most advanced vacuum microelectronics devices are based upon field emission processes [3]. Recent measurements of thinfilm field-emitting cathodes ("Spindt" cathode [4]) produced current densities $\approx 1 \mathrm{kA} / \mathrm{cm}^{2}$ and transconductance $g$ per unit cathode area $\approx 100 \mathrm{~S} / \mathrm{cm}^{2}(g=$ $\left.\left(\Delta I_{A} / \Delta V_{g}\right)_{V_{A}}\right)$ for sample 44-234-2Q at SRI. This exceeds the performance of gridded thermionic cathodes by 10 and 100 , respectively. The application of such cathode structures to electron-beam sources and RF amplification is therefore potentially useful. A three-terminal amplifier or triode is the simplest device type for this latter purpose. Therefore, a triode amplifier geometry based upon the "Spindt" cathode is discussed in this paper.

The cathode structure being simulated is based upon the structures from SRI currently being used in experiments at NRL [5] and SRI [6]. The Spindt gated cathode array [4] consists of many conical field emitter tips each with its own self-aligned surrounding gate. The addition of an anode surface forms a triode. Azimuthal symmetry about the axis of the cone is assumed. Geometrical factors, for example, tip sharpness, tip height, cone half-angle, and gate hole radius, which influence the field may be varied by control in processing steps. The geometry of the unit cell is shown in Fig. 1. The emitted current depends upon

Manuscript received September 25, 1992; revised January 11, 1993. This work was supported by the Naval Research Laboratory and the Office of Naval Research. The revicw of this paper was arranged by Associate Editor I. Brodie.

The author is with the Naval Research Laboratory, Washington, DC 20375 .

IEEE Log Number 9208065.

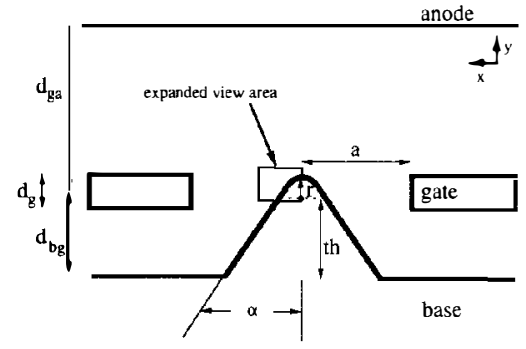

Fig. 1. Unit cell geometry of field emission microtriode.

the electric field in the vicinity of the small radius of curvature tip.

Triode device performance has been studied by simulating a unit cell consisting of a single emitter tip, gate, and anode. The emission current for a set of applied voltages from such a cell depends upon the work function, emission area, and tip geometry. A study of the variations in tip geometry while assuming an average value for work function, and allowing the entire tip to emit, may provide a sufficient understanding of tip performance for the purpose of microtriode design. The extrapolation of performance based upon the unit cell neglects several factors of importance in the real device, such as the interelectrode capacitances and its effect upon the frequency limits. Because the dependence of the frequency limit upon the transit time is negligible, operation of a field-emission microtriode at frequencies above those currently achievable with solid-state devices is of interest. The modeling development includes the time dynamic evolution of an RF cycle showing beam propagation, and current versus time at selected planes.

\section{Simulation Method}

A space-charge-free model has previously been used by Fecney et al. [7] to provide the variation of electric field in a triode configuration as a function of tip radius, cone angle, and tip height. A diode structure has been studied by Marcus et al. [8] using the electron optics simulation code SIMION 4.0. Emission currents, which depend upon tip radius, tip height, and cone angle, were calculated and a good discussion of tip heating effects was included.

To provide a more complete model of the device performance based upon the unit cell approach, the simulations used a modified version of the widely used MAGIC particle-in-cell simulation code [9] on the NRL Cray $\mathrm{X}$-MP computer. The program is a two-dimensional, 


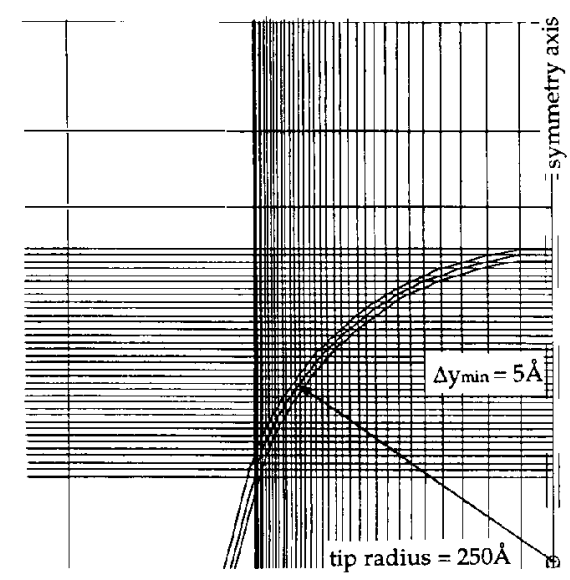

Fig. 2. Simulation emission surface. Note the use of a variable spatial mesh and nonconformal emission surfaces. Minimum $\Delta y \approx 5 \AA$ and minimum $\Delta x \approx 1.4 \dot{\AA}$ for the $250-\AA$ tip radius shown.

finite-difference time-domain code for the self-consistent simulation of the interaction of charged particles and electromagnetic fields. The full set of Maxwell equations and the complete Lorentz force equation is utilized. Spacecharge effects are automatically included. Boundary conditions include conductors and electron emitters. which need not be conformal to the grid mesh. A variable spatial mesh, shown in Fig. 2, resolved the tip geometry while using a manageable number of mesh points for the simulation of the entire unit cell without piecing solutions together. Spatial resolution was varied from below 5 to 50 $\AA$ with checks for consistency in going to the larger meshes which were used for the RF dynamic simulations. As can be seen in Fig. 2, the emission surface was approximated by patching together conical sections, or facets, to approximate a hemispherical surface with small surface roughness, blending smoothly to the principal conical support. The superposition of additional microstructures or surface roughness was unnecessary for the comparison of the unit ccll with the triodc charactcrization of interest.

A Fowler-Nordheim emission [10] module was developed and inserted into the MAGIC code to model electron field emission from the surface. The current $J$ was calculated from

$$
J=\frac{A E^{2}}{\phi t^{2}} \exp \left(\frac{-\boldsymbol{B} v(y) \phi^{3 / 2}}{E}\right)
$$

where $A=1.5414 \times 10^{-6}, E=$ the normal component of the electric field at the emitter surface, $\phi=$ the work function of the emitter, taken as $4.35 \mathrm{eV}$ for Mo, $B=$ $6.8308 \times 10^{9}, t^{2}=1.1$, an approximation, and $v(y)=$ $0.95-y^{2}$ with $y=3.79 \times 10^{-5} \times E^{1 / 2} / \phi$ in SI units. Initially, the Green's function solutions of the Laplace equation are computed for the given geometry. The field along the emitter surface is determined by superposition of the contribution from each conductor. The emitted charge is governed by the Fowler-Nordheim rclation ap- plied locally at each cell along the emitter. Although current could be emitted from both the spherical and conical portions of the tip, the contribution from the sides of the cone was several orders of magnitude down and could be neglected. The electric field at the surface is then adjusted by Gauss's law to allow for field perturbation due to the emitted charge. The simulation proceeded, after this module, by pushing the particles, updating the fields, and then self-consistently cvolving in time to determine the new emitted current required using the updated fields; this process continuing for the specified number of loops.

\section{Geometrical Dependence of Emission Current}

Unit cell parameters were varied about the nominal dimensions of current experiments at NRL, which are (Fig. 1): the base-gate distance, $d_{b g}=1.15 \mu \mathrm{m}$; gate thickness, $d_{g}=0.3 \mu \mathrm{m}$; and a large gate-anode distance of $d_{g a}=$ $100 \mu \mathrm{m}$. Tip-to-tip distance was $5.0 \mu \mathrm{m}$, with the effects of adjacent tips allowed in the unit cell by the application of a mirror simulation boundary. Four other geometrical dimensions were also varied individually about a base case with the tip height, $t_{h}=1.25 \mu \mathrm{m}$; a tip radius, $r=250$ $\AA$; gate hole radius, $a=0.55 \mu \mathrm{m}$; and tip half-angle, $\alpha$ $=15^{\circ}$. The work function was taken as that of clean Mo, $4.35 \mathrm{eV}$ [11]. For the purposes of simulation, the tip height was defined as the distance from the base plane of the emitting tip to the center of curvature of the spherical segment. This spherical surface was blended smoothly to the conical tip sides.

The match to experimental data was good for samples studied at NRL. The data for the sample denoted 405A [5] has been previously published and is shown for a typical comparison. A one parameter fit on tip radius gave the results shown in Fig. 3 for $r=185 \AA$ which is within the error limits for the electron microscope used [12]. Consequently, an additional tip structure was not imposed upon the simulation as has been done by Herrmannsfeldt et al. [13] and Rosengreen et al. [14]. Due to the extreme sensitivity of the emitted current upon the tip radius, a variation of tip radius by itself can account for current experimental data in the parameter regime of interest to RF power generation. The effects of geometrical variations in the simulated unit cell are described below.

This strong dependence of the emitted current upon the tip radius is shown in Fig. 4 for variation from 100 to 400 $\AA$. A spherical diode has a $1 / r$ variation in electric field at the inner surface which translates into a large difference in emitted current for a Fowler-Nordheim emitter over this range of emitter radii. In the unit cell geometry chosen, for a gate voltage of $140 \mathrm{~V}$, the simulation exhibited a variation of scven orders of magnitude in emitted current! For a 90-V gate voltage, almost eleven orders of magnitude current variation was noted. As the tip radius was decreased, the difference in current at the selected gate voltages became much smaller. For the $140-\mathrm{V}$ casc, the dependency on tip radius may be approximated as $10^{7}$ $\mathrm{A} / 300 \AA=3.3 \times 10^{4} \mathrm{~A} / \AA$. 


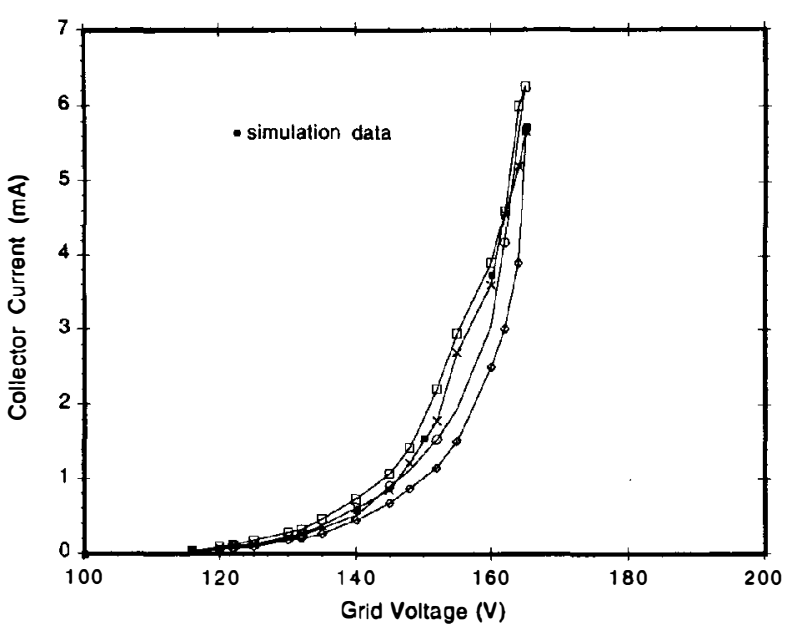

Fig. 3. Current-voltage transfer characteristic for sample $405 \mathrm{~A}$ and simulation. Simulation data are indicated by solid squares.

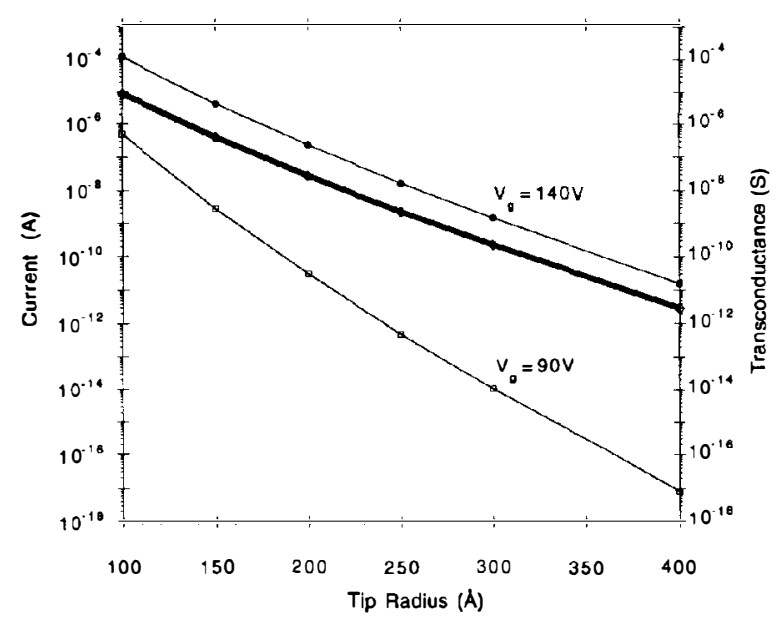

Fig. 4. Emitted current as a function of tip radius at $90 \mathrm{~V}$ (lower curve) and $140 \mathrm{~V}$ (upper curve). Transconductance at $140 \mathrm{~V}$ is indicated in bold.

The electric field enhancement may be defined as the peak electric field at the tip divided by the electric field for parallel plates with separation equal to the base-gate distance. Fig. 5 shows the very large enhancement with decreased tip radius similar to the results of Feeney et al. [7].

The variation of emitted current with tip half-angle is not so dramatic. As seen in Fig. 6, for the standard case of $140 \mathrm{~V}$, changing the tip half-angle $15^{\circ}$ to $1^{\circ}$ changed the current by only one order of magnitude. At the somewhat extreme half-angle of $45^{\circ}$, the emitted current is five orders of magnitude smaller than the $1^{\circ}$ case. The dependency of current on half-angle is approximatcly linear between $1^{\circ}$ and $30^{\circ}$ with a slope of $2 \times 10^{3} \mathrm{~A} /$ degree. The electric field enhancement exhibits approximately linear behavior over a wide range of tip half-angle as seen in Fig. 7.

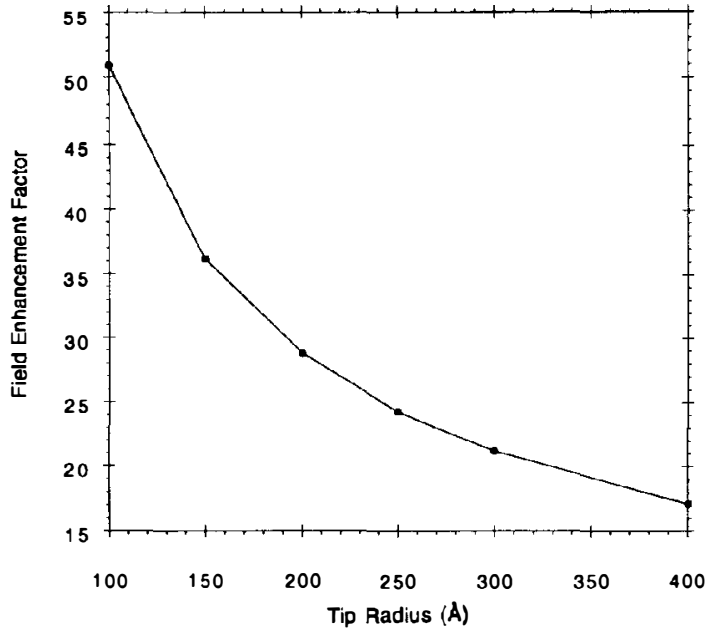

Fig. 5. Electric field enhancement at $140 \mathrm{~V}$ as a function of tip radius.

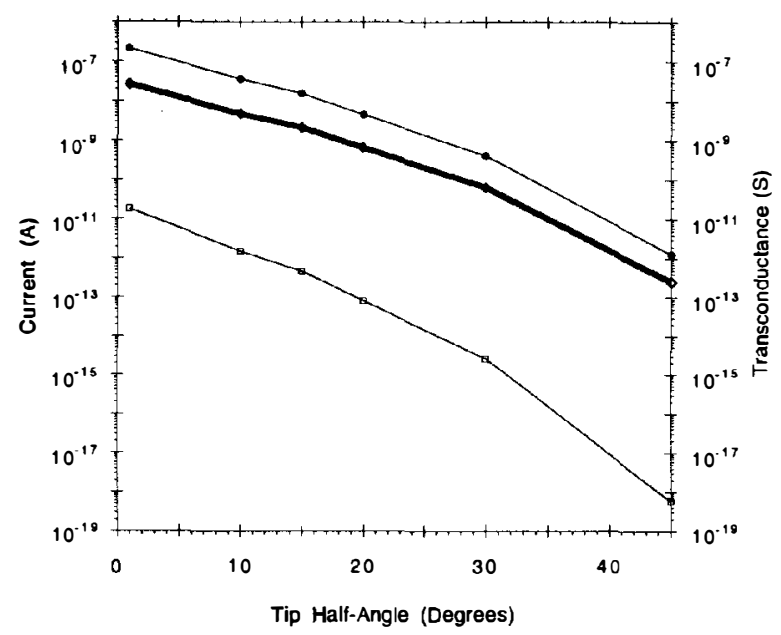

Fig. 6. Emitted current as a function of tip half-angle at $90 \mathrm{~V}$ (lower curve) and $140 \mathrm{~V}$ (upper curve). Transconductance at $140 \mathrm{~V}$ is indicated in bold.

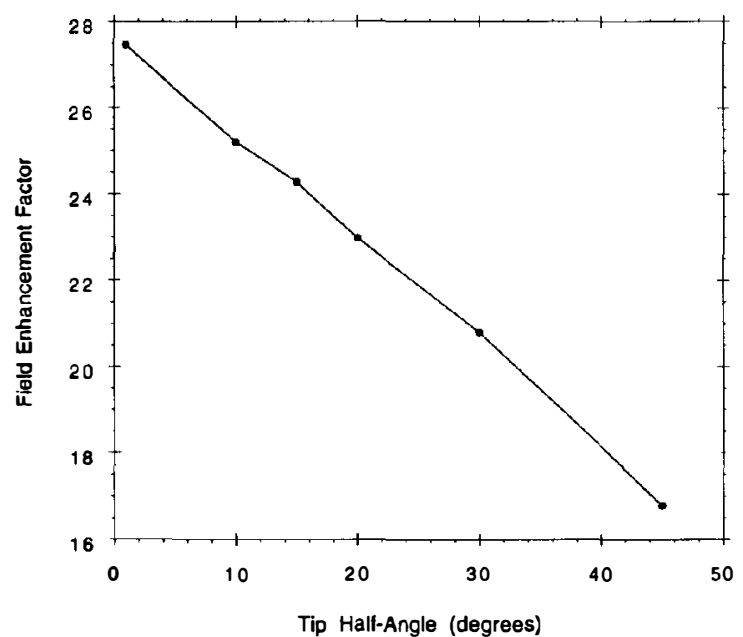

Fig. 7. Electric field enhancement at $140 \mathrm{~V}$ as a function of tip half-angle. 


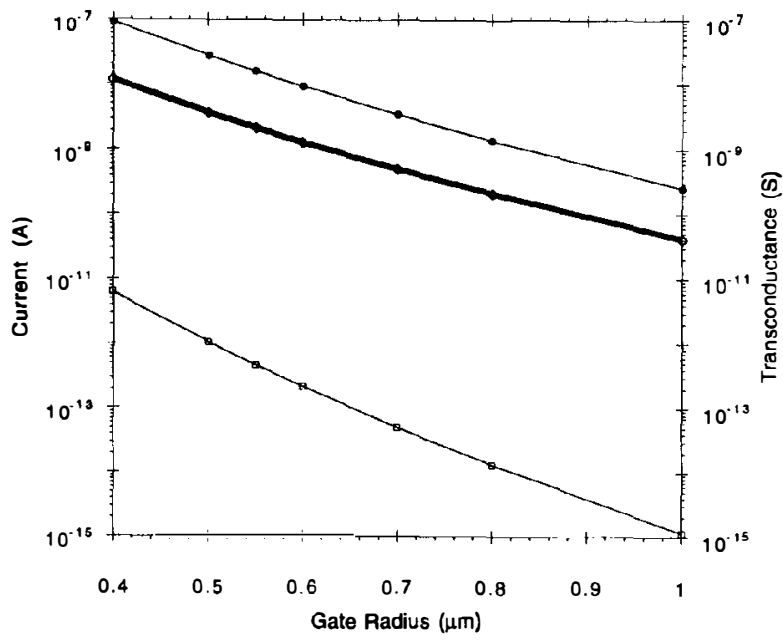

Fig. 8. Emitted current as a function of gate hole radius at $90 \mathrm{~V}$ (lower curve) and $140 \mathrm{~V}$ (upper curve). Transconductance at $140 \mathrm{~V}$ is indicated in bold.

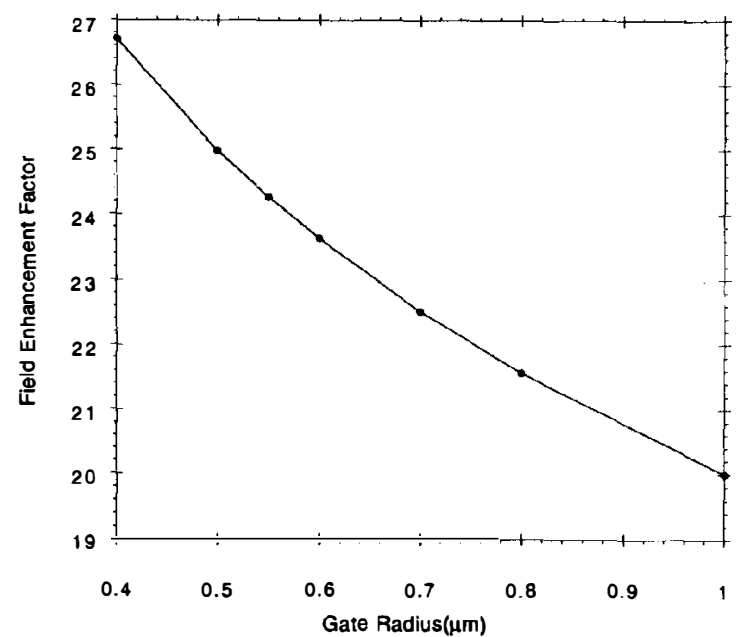

Fig. 9. Electric field enhancement at $140 \mathrm{~V}$ as a function of gate hole radius.

The dependence of emitted current upon gate hole radius is shown in Fig. 8. For the radii of interest $(0.4$ to $0.6 \mu \mathrm{m}$ ) only about one order of magnitude variation in the emitted current was observed for a $140-\mathrm{V}$ gate voltage. The current sensitivity to gate radius is $5 \times 10^{1}$ $\mathrm{A} / \mu \mathrm{m}$. The electric field is enhanced only on the ordcr of $10 \%$ in going from 0.55 to $0.4 \mu \mathrm{m}$ as shown in Fig. 9 .

A variation of the "tip height" within the gate thickness (Fig. 10) varied by only about two orders of magnitude with the smallest tip height providing the most current in the chosen geometry. The tip is at least as tall at the gate bottom to avoid gate interception of the current. The current dependence on tip height $4 \times 10^{2} \mathrm{~A} / \mu \mathrm{m}$ is relatively weak with a somewhat different dependence than that seen by Feeney et al. [7], probably due to their much larger gate hole diameter.

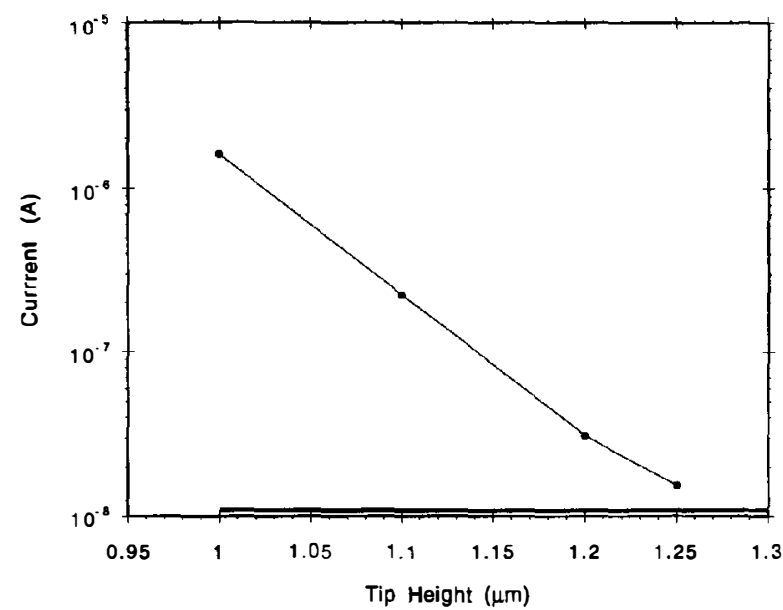

Fig. 10. Emitted current as a function of tip hcight at $140 \mathrm{~V}$. The gate location is indicated by the bold line at the bottom.

\section{Beam Characterization}

A new approach for the creation and acceleration of a high-brightness, high-quality electron beam with a compact form factor of intercst for applications such as freeelectron lasers, accelerators, and gyrotrons may be possible with field emission arrays. The use of field emission arrays in electron-beam sources is of interest due to their small size ( $\approx 10 \mu \mathrm{m}$ per unit cell) and high current density $\left(\approx 1 \mathrm{kA} / \mathrm{cm}^{2}\right)$. Their robustness in such a hostile environment would need to be addressed. Photocathodes have recently been utilized effectively: however, an external laser driver is required to address concerns of efficiency and system complexity. Roberson [15] has defined a freeelectron laser beam quality as the current density divided by the total relative energy spread, $B_{Q}=J /\left(\Delta \gamma_{z} / \gamma\right)$ where $\gamma$ is the relativistic energy to rest energy ratio. The extent to which the beam-wave interaction sees the beam as monoenergetic may be thus quantified. This quantity is related to the normalized brightness, where the spread in energy is due to the emittance [16], $B_{n}=J / 2 \pi\left(\Delta \gamma_{z} / \gamma\right)_{\epsilon}$ $=I / \pi^{2} \epsilon_{n}^{2}$.

A look at a typical unit cell can provide some of the required beam characterization. Cell-to-cell uniformity may be an important factor to determine overall beam quality; however, it is not addressed here. Consider a typical unit triode cell with a base-gate distance $d_{b q}=1.15$ $\mu \mathrm{m}$, a gate thickness $d_{g}=0.3 \mu \mathrm{m}$, a closer gate-anode distance of $d_{g a}=5.0 \mu \mathrm{m}$, tip height $t_{h}=1.25 \mu \mathrm{m}$, a tip radius $r=150 \AA$, gate hole radius $a=0.55 \mu \mathrm{m}$, and tip half-angle $\alpha=15^{\circ}$, a tip current of $4.1 \times 10^{-5} \mathrm{~A}$ was emitted. More than $97 \%$ of this current was contained within a radius $r=2.44 \times 10^{-6} \mathrm{~m}$ at the plane $z=6.15$ $\times 10^{-6} \mathrm{~m}$ yielding a normalized emittance $\epsilon_{n}=3.97 \times$ $10^{-8} \mathrm{mrad}$. The normalized brightness for this unfocused beam at this plane is $2.63 \times 10^{9} \mathrm{~A} /(\mathrm{mrad})^{2}$. Approximating the current density by a uniform beam yields $J=$ $2.2 \times 10^{6} \mathrm{~A} / \mathrm{m}^{2}$. As the space charge is negligible for the chosen case, the emittance is the principal cause of 
energy spread from the unit cmitting cell. The beam quality may be taken as $B_{Q}=2 \pi B_{n}=1.65 \times 10^{10} \mathrm{~A} / \mathrm{m}^{2}$.

An electrostatic focusing electrode above the gate hole may significantly improve these unit cell numbers by controlling emittance spread. With this current confined with the same energy spread to a beam radius of $0.7 \mu \mathrm{m}$, normalized brightness would be increased by more than 12 times to $3.2 \times 10^{10} \mathrm{~A} /(\mathrm{mrad})^{2}$. The use of an array of unit cells suggests that a beam diameter per tip comparable to the tip-to-tip spacing is a more useful approximation. Emittance growth due to the combining of beams from multiple cells [17] would need to be examined for any realistic system.

\section{Triode Characterization}

Several figures of merit may be calculated for RF triodes. In order to achieve high gain in an amplifier configuration, the transconductance, $g=\left(\Delta I_{A} / \Delta V_{g}\right)_{V_{A}}$, needs to be high. This permits a large amount of current to be switched with small voltages on the grid. The variation of transconductance with geometrical parameters is of interest in practical design and is thus shown in Figs. 4, 6, and 8 for a gate voltage of $V_{g}=140 \mathrm{~V}$. For the standard geometrical values used herein, the transconductances for tips of radii 250 and $100 \AA$ are $g=0.00212 \mu \mathrm{S}$ and $g=$ $7.75 \mu \mathrm{S}$, respectively. Often the transconductance is desired per unit area. For the $100 \AA$ case, $g=7.75 \mu \mathrm{S} /(5$ $\left.\times 10^{-6} \mathrm{~m}\right)^{2}=3.1 \times 10^{5} \mathrm{~S} / \mathrm{m}^{2}$. The corresponding cutoff frequencies $\left(f_{t}=g / 2 \pi C_{g}\right)$ for these tips are $f_{t}=1.75$ $\mathrm{MHz}$ and $f_{t}=6.39 \mathrm{GHz}$, respectively, for a gate capacitance $0.193 \mathrm{fF}$.

High transconductances have been achieved to date, but with high currents. In order to avoid difficulties with thermal dissipation and tip disruption, one may wish to operate with a more modest current per tip of tens of microamperes. High transconductance then needs to be achieved by greater sensitivity on gate voltage. Geometrical dependencies of the gate voltage and transconductance are therefore calculated for a fixed current of $10 \mu \mathrm{A}$ by application of a Fowler-Nordhcim emission formula fitted to the previous 140- and 90-V curves. In Fig. 11, the transconductance per tip increased by almost a factor of two with a decrease in radius from 250 to $100 \AA$. The transconductance is approximately linear with tip half-angle, as seen in Fig. 12. The second derivative of the required gate voltage as a function of tip half-angle remains positive. The gate voltage departs only slightly from linear behavior as a function of gate radius (Fig. 13).

$\mathrm{RF}$ amplifier operation using an emitter array is desired. The current-voltage characteristics of a 150 - $\AA$ triode are shown in Fig. 14. The emitted current is shown as a function of the anode voltage for various gate voltages. The extreme flatness of the characteristics for $V_{A}$ just a few tens of volts larger than $V_{g}$ indicates a high output impedance. For a $100-\mathrm{k} \Omega$ load resistance and 400 -V bias source, a load line for a 100-tip array may be

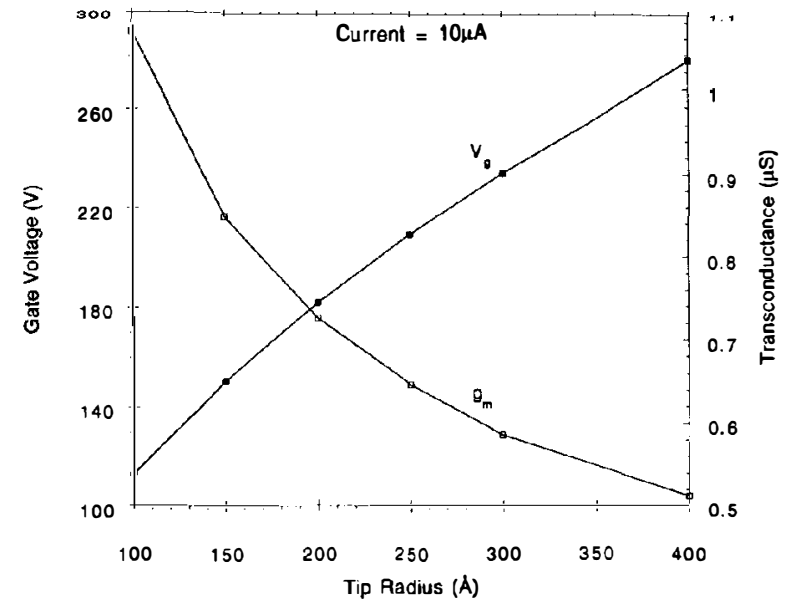

Fig. 11. Gate voltage and transconductance at $10 \mu \mathrm{A}$ as a function of tip radius.

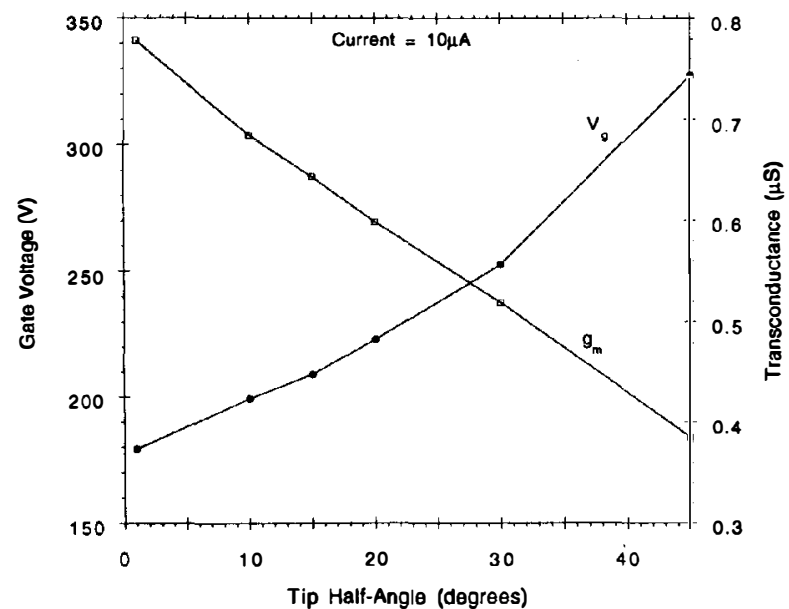

Fig. 12. Gate voltage and transconductance at $10 \mu \mathrm{A}$ as a function of tip half-angle.

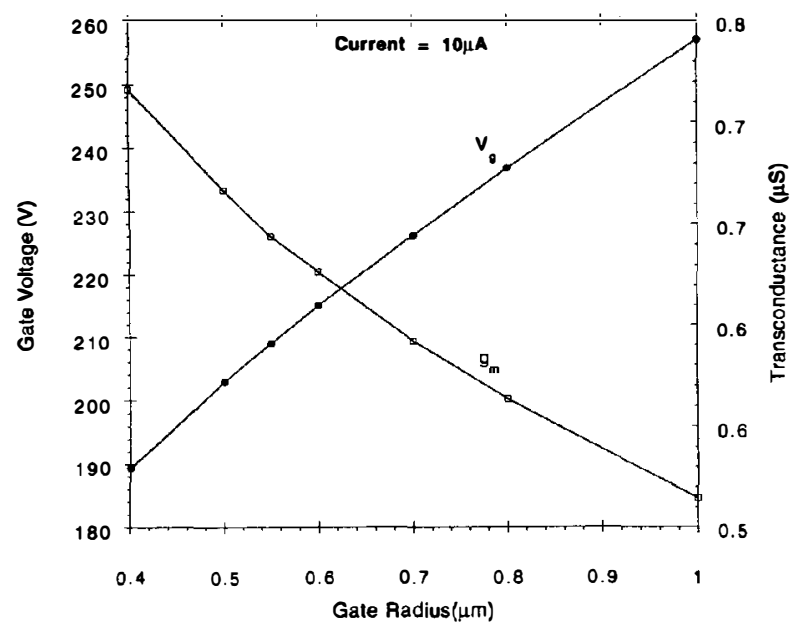

Fig. 13. Gate voltage and transconductance at $10 \mu \mathrm{A}$ as a function of gate hole radius. 


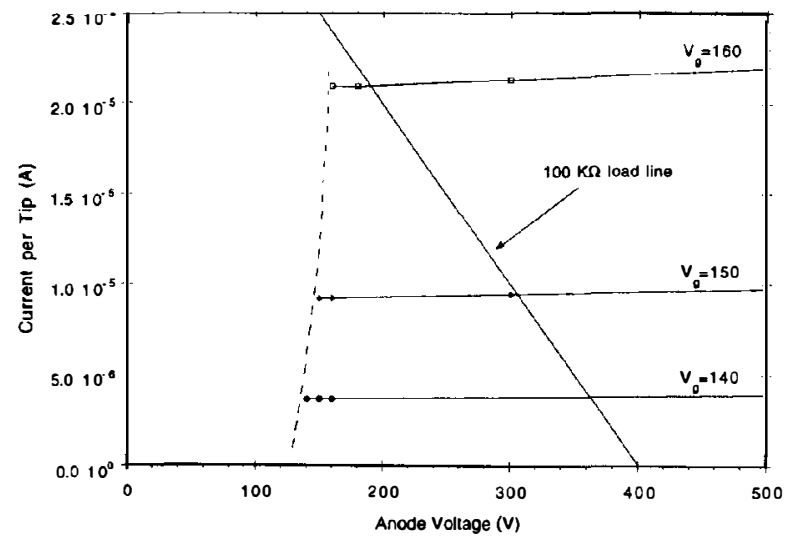

Fig. 14. Emitted current-anode voltage characteristics at varied gate voltages. An operating regime between a grid voltage of 150 and $160 \mathrm{~V}$ along a $100-\mathrm{k} \Omega$ load line biased at $400 \mathrm{~V}$ for a 100 -tip array as discussed in the text.

constructed as shown in Fig. 14. The simulated triode, operating along this load line between grid voltages of 150 and $160 \mathrm{~V}$, has a voltage gain of $21 \mathrm{~dB}$. The 100-tip triode circuit has a class A (small signal) efficiency of $2.7 \%$ with $0.017-\mathrm{W}$ output power.

To examine the dynamic characteristics of a high-performance triode, a simulation using approximately the geometry of the 44-234-2Q structure was performed. As properties (such as power deposition) associated with the anode are of interest, the gate-anode distance was decreased to a value of $d_{g a}=1.65 \mu \mathrm{m}$, approximately three times the gate hole radius. A planar anode was used. Other applicable dimensions are: a base-gate distance of $d_{b g}=$ $1.15 \mu \mathrm{m}$, a gate thickness of $d_{g}=0.3 \mu \mathrm{m}$, an effective tip-to-tip distance of $3.0 \mu \mathrm{m}$, a tip height of $t_{h}=1.25$ $\mu \mathrm{m}$, a tip radius of $r=235 \AA$, a gate hole radius of $a=$ $0.50 \mu \mathrm{m}$, and a tip half-angle of $\alpha=15^{\circ}$. With an anodetip voltage $V_{A}=500 \mathrm{~V}$ and a sinusoidal variation of the gate-tip voltage with an amplitude of $V_{g \text { Max }}=212 \mathrm{~V}$, the beam trajectories and anode power deposition were examined as a function of time. Fig. 15 shows the behavior of the electron current emanating from the tip as the sinusoidal voltage is turned on. There is one streamline from each facet of the emitting surface. It is important to emphasize that each streamline denotes the flow of electrons from a particular facet and does not indicate the magnitude of the current along the streamline. A $1-\mathrm{THz}$ simulation frequency was used to accentuate any transit-time effects and to keep computational costs down. The current flux through the gate top plane and to the anode plane is shown in Fig. 16. There is no discernible distortion of the waveform. Of particular interest is the anode power deposition, which is shown in Fig. 17 for different times as a function of distance along the anode from the tip axis. The peak power of $4.00 \times 10^{5} \mathrm{~W} / \mathrm{m}^{2}$ is significantly large. The reduction of tip radius to $100 \AA$ would increase the current by three orders of magnitude resulting in a peak power in excess of $10^{8} \mathrm{~W} / \mathrm{m}^{2}$ which is well
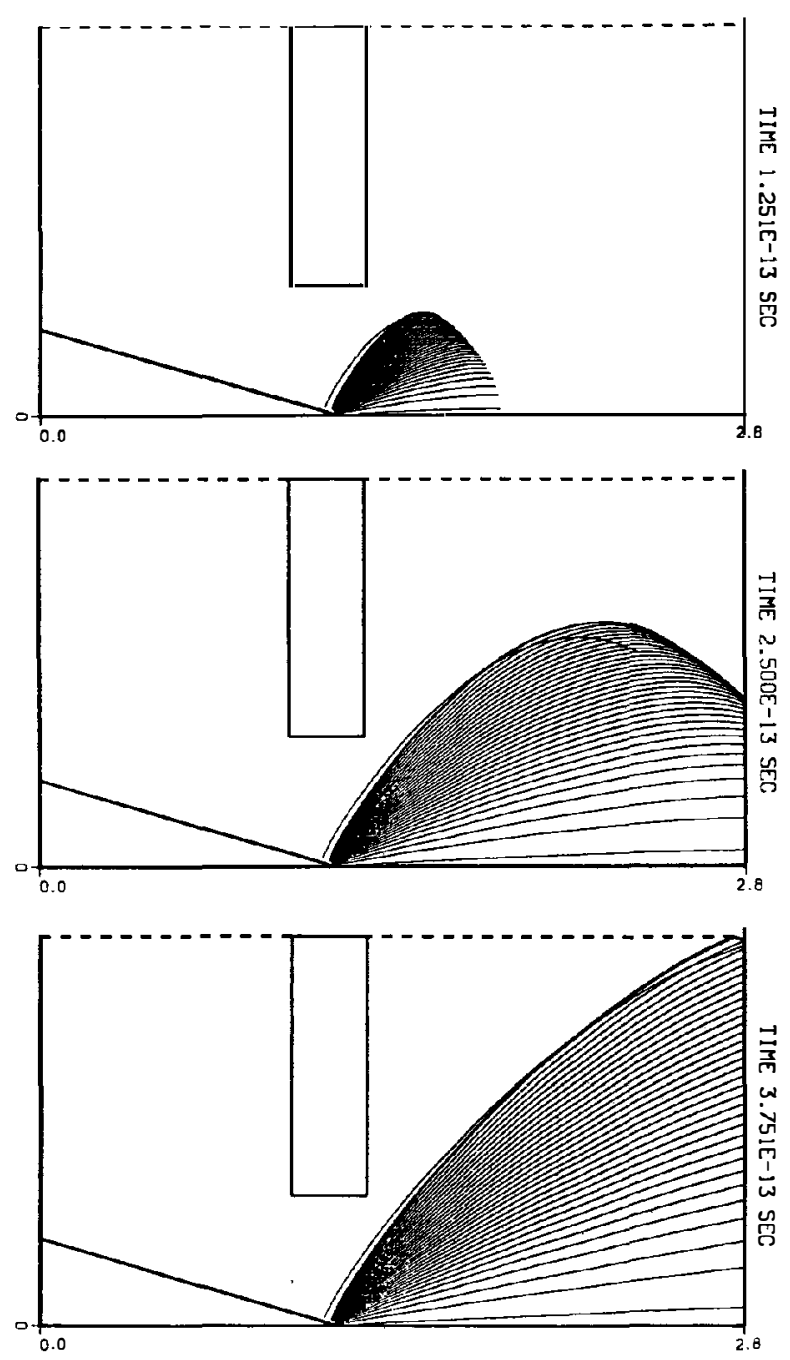

Fig. 15. Electron beam streamlines during the application of a sinusoidal gate voltage at $1 \mathrm{THz}$.

beyond the limits of known materials and indicates the necessity of alternate geometries and materials for anode construction.

\section{Vi. Discussion and Conclusions}

The dependence of emitted current on 1) tip height, 2) tip radius, 3) tip half-angle, and 4) gate hole radius has been determined and shows an extreme variation with tip radius in the 100 - to 400 - $\AA$ range. Device characteristics of transconductance, cutoff frequency, gain, and efficiency have been calculated from the unit cell and are indicative of the possibility of useful triode devices in the few tens of gigahertz range if the parasitic capacitances and resistive losses can be minimized. With thousands of tips per emitter array, the total transconductance can be quite large (e.g., $g=7.75 \mu \mathrm{S} \times 10000$ tips). The nor- 


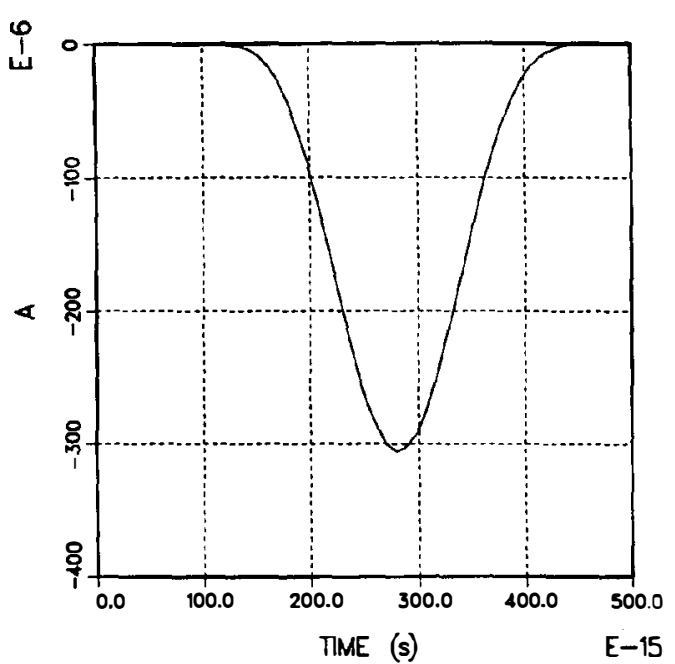

(a)

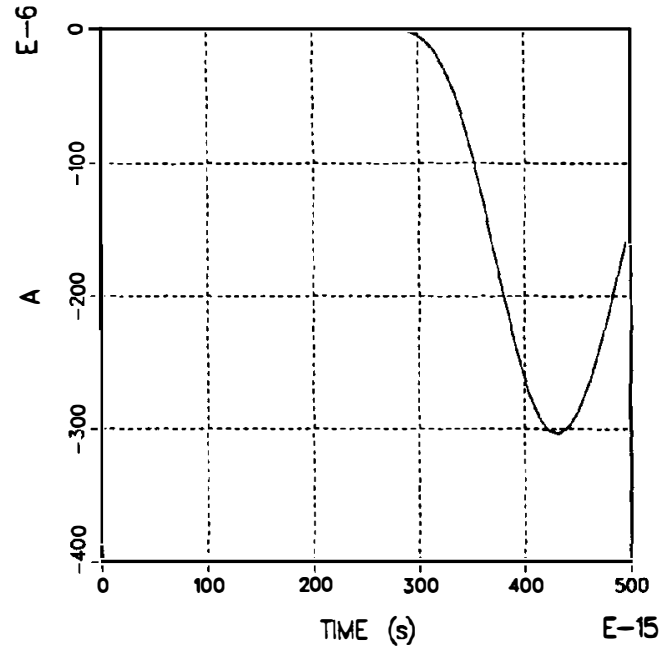

(b)

Fig. 16. Current $(\mu \mathrm{A})$ flux as a function of time through planes at the top of the gate (a) and just below the anode (b).
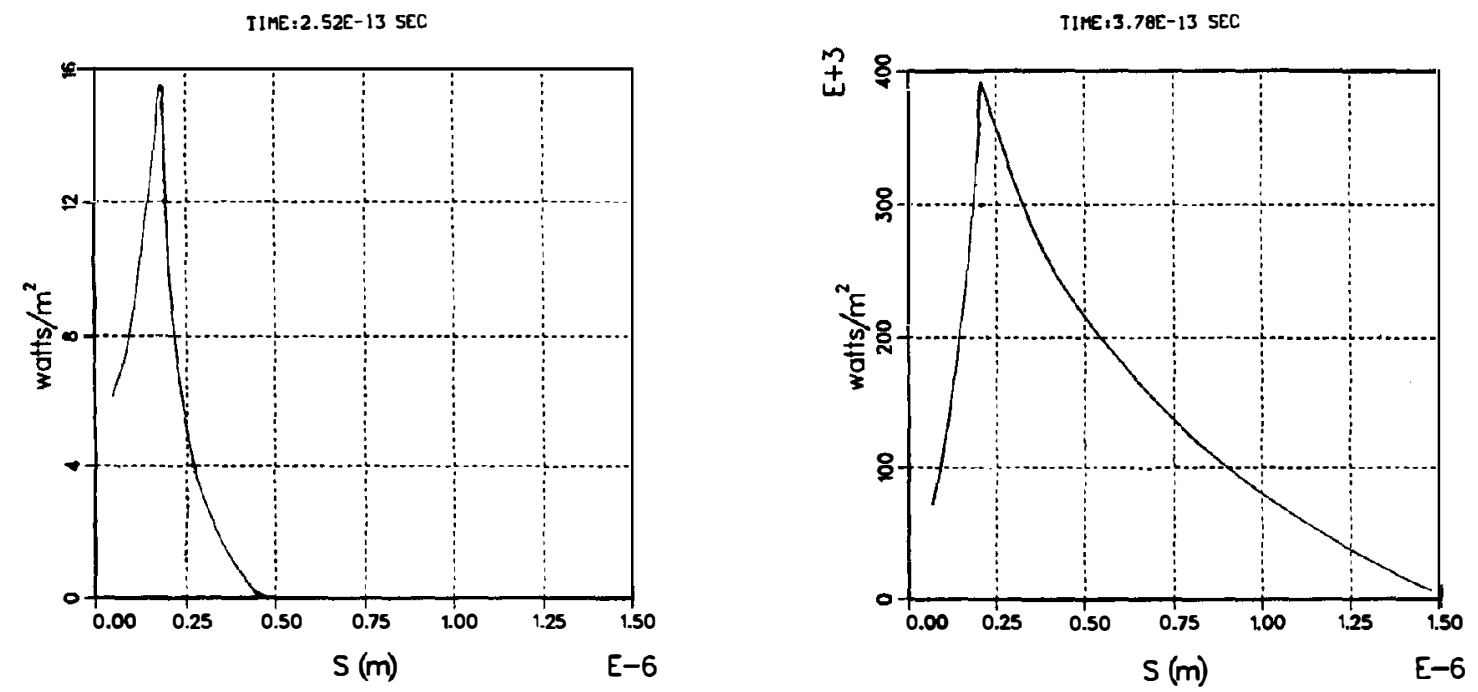

Fig. 17. Envelope of anode power deposition as a function of radius at varied times

malized beam brightness and current densities calculated for the unit cell are similar to pulse line and RF linac beam sources currently in use. At only slightly below photocathode beam brightness, without the laser requirements, similar focused structures could be considered for compact beam source requirements.

The dynamic behavior in a microtriode has been examined including beam trajectory spreading, anode power deposition, and transit-time current distortion. The transittime distortion is seen to be negligible and the effect of space charge on the spreading of the beam is small. The anode power deposition is seen to present such a major problem that alternative geometries and materials need to be examined for this application.

\section{ACKNOWLEDGMENT}

The author wishes to gratefully acknowledge helpful discussions with H. Gray, P. M. Phillips, A. K. Ganguly, K. L. Jensen, J. P. Calame, and R. K. Parker.

\section{REFERENCES}

[1] K. Shoulders, Advances in Computers, F. L. Alt, Ed. New York: Academic Press, 1961, pp. 135-293.

[2] R. Green, H. Gray, and G. Campisi. "Vacuum integrated circuits,' in IEDM Tech. Dig.. 1985, p. 172.

[3] T. Utsumi, "Vacuum microelectronics: What's new and exciting," presented at the 3rd Int. Conf. on Vacuum Microelectronics, Monterey, CA, 1990.

[4] C. A. Spindt, I. Brodie, L. Humphrey, and E. R. Westerberg, "Phys- 
ical properties "r thin-film emission cathodes with molybdenum

15] R. E. Neidert, P. M. Phillips, S. T. Smith, and C. A. Spindt, "Field emission triodes," IEEE Trans. Electron Devices, vol. 38, p. 661, 1991 .

[6] C. E. Holland, A. Rosengreen, and C. A. Spindt, "A study of field emission microtriodes," presented at the 3rd Int. Conf. on Vacuum Microelectronics, Montcrey, CA, 1990.

[7] R. K. Fceney, J. K. Cochran, D. N. Hill, and A. T. Chapman, "A mathematical model to predict optimum geometry of the elements of a field emission array cathode," Instr. Phys. Conf., vol. 99, 1989.

[8] R. B. Marcus, K. K. Chin, Y. Yuan, H. Wang, and W. N. Carr, "Simulation and design of field emitters," IEEE Trans. Filectron Devices, vol. 37, pp. 1545-1550. 1990

[9] B. Goplen, L. Ludeking, J. McDonald, G. Warren, and R. Worl, MAGIC User's Manual, Mission Research Corp., MRC/WDC-R-184, 1988.

[10] R. H. Fowler and I. W. Nordheim, "Electron emission in intense electric fields," Proc. Roy. Soc., vol. A119, p. 173, 1928.

[11] G. A. Haas, American Institute of Physics Handbook, 3rd ed. New York: McGraw-Hill, 1972, pp. 9-172.
[12] P. M. Phillips, private communication.

[13] W B. Hernnannsfeldt, R. Becker, I. Brodie, A. Rosengreen, and C. A. Spindt, "High-resolution simulation of field emission," Nuclear Inst. Methods in Phys. Res., vol. A298, 1990.

[14] A. Rosengreen, 1. Brodie, C. A. Spindt, and D. E. Holland, "Modeling of the Spindt field emitter cathode," in Abstracts 3rd Int. Vac' uum Microelectronics Conf. (Monterey, CA), 1990.

[15] C. W. Roberson, "Free-electron laser beam quality," IEEE J. Quan tum Electron., vol. QE-21, p. 860, 1985

[16] J. D. Lawson, The Physics of Charged-Particle Beams. London, UK: Oxford Univ. Press, 1977, p. 178

[17] C. M. Celata et al., "Transverse combining of nonrelativistic beams in a multiple beam induction Linac," in 1987 JEEE Particle Accelerator Conf. (Washington, DC), p. 1167

Ernest G. Zaidman, photograph and biography not available at the time of publication. 